\title{
Temperature Induced Instabilities in Macro-bend Fiber Based Wavelength Measurement Systems
}

\author{
Ginu Rajan \\ Technological University Dublin, ginu.rajan@tudublin.ie \\ Yuliya Semenova \\ Technological University Dublin, yuliya.semenova@tudublin.ie \\ Pengfei Wang \\ Technological University Dublin, pengfei.wang@tudublin.ie
}

See next page for additional authors

Follow this and additional works at: https://arrow.tudublin.ie/engscheceart

Part of the Electrical and Computer Engineering Commons

\section{Recommended Citation}

Rajan, G. et al (2009) Temperature induced instabilities in macro-bend fiber based wavelength measurement systems. Journal of Lightwave Technology, Vol. 27, no. 10, pp.1355-1361. D0I:10.1109/ JLT.2009.2014081

This Article is brought to you for free and open access by the School of Electrical and Electronic Engineering at ARROW@TU Dublin. It has been accepted for inclusion in Articles by an authorized administrator of ARROW@TU Dublin. For more information, please contact arrow.admin@tudublin.ie, aisling.coyne@tudublin.ie, gerard.connolly@tudublin.ie.

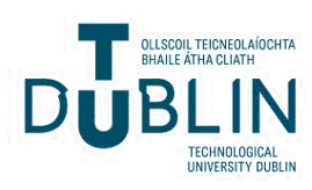




\section{Authors}

Ginu Rajan, Yuliya Semenova, Pengfei Wang, and Gerald Farrell

This article is available at ARROW@TU Dublin: https://arrow.tudublin.ie/engscheceart/52 


\title{
Temperature-Induced Instabilities in Macro-Bend Fiber Based Wavelength Measurement Systems
}

\author{
Ginu Rajan, Yuliya Semenova, Pengfei Wang, and Gerald Farrell
}

\begin{abstract}
An investigation of temperature-induced instabilities in a wavelength measurement system based on macro-bend fiber filter used in the ratiometric scheme are presented. Two wavelength measurement systems based on macro-bend fiber, a standard low bend loss single-mode fiber filter based system and a high bend loss fiber filter based system are considered. In the case of a low bend loss fiber filter based system, the oscillatory variation in the ratio response with temperature and the difference in the temperature-induced ratio variation at different wavelengths makes the temperature corrected calibration of the system unfeasible, which is essential for precise wavelength measurements. The high bend loss fiber filter based system is more sensitive to temperature than the low bend loss fiber filter based system, but critically for the high bend loss fiber filter based system the ratio has a linear variation with temperature, which can be corrected by a suitable temperature calibration process. Comprehensive studies on the temperature dependence of both the macro-bend fiber filter system and its effect on wavelength measurements are presented in this paper.
\end{abstract}

Index Terms-Macro-bend fiber filters, temperature dependence, wavelength measurements.

\section{INTRODUCTION}

A MACRO-BEND fiber edge filter based ratiometric wavelength measurement system offers high-speed wavelength measurement with high resolution and the advantage of low fabrication complexity [1]. This low-cost wavelength measurement system is attractive for use as an interrogation system for an array of FBG's due to its wide wavelength range [2]. However the accuracy of the system over a wide wavelength range is a concern. One of the main factors introducing inaccuracies to the measured wavelength is ambient temperature variation. It has been proved earlier that temperature has a significant influence on the bend loss of macro-bend fibers [3]-[6] and therefore on the ratio of the system and the measured wavelength. Commonly a ratiometric system is calibrated and the ratio response is obtained at a fixed temperature. However a subsequent change in ambient temperature for the system can alter the ratio response and can lead to inaccurate wavelength measurements.

In macro-bend fiber edge filters, an absorption layer is applied to the buffer coating to absorb the whispering gallery modes (WGM), which are inherent in bent single-mode fibers. Macrobend fiber edge filters can be made from standard single-mode

Manuscript received June 30, 2008; revised October 13, 2008. First published April 24, 2009; current version published May 08, 2009.

The authors are with Photonics Research Centre, School of Electronic and Communications Engineering, Dublin Institute of Technology, Dublin 8, Ireland (ginu.rajan@dit.ie).

Digital Object Identifier 10.1109/JLT.2009.2014081 fiber (such as SMF28) [1] and a high bend loss fiber (such as 1060XP) [7]. For fiber filters based on a single-mode fiber such as SMF28, which has a dual coating layer, even with the absorption layer a low level of residual internal reflection exists at the cladding-primary coating boundary, which leads to temperature-induced variations in the bend loss. For fiber filters based on bend sensitive fibers like 1060XP, the absorption layer is applied directly to the cladding providing a linear variation in ratio with temperature. In this paper a detailed investigation of the temperature dependence of a ratiometric system based on both an SMF28 fiber filter and 1060XP fiber filter and its effect on wavelength measurements are presented. In Section II the background to the temperature dependence of macro-bend single-mode fibers is presented. An experimental setup to measure the temperature-induced ratio and wavelength variation is described in Section III. Results, discussion and a comparison of temperature-induced wavelength variation for both fiber types are presented in Section IV.

\section{BACKGROUND}

When a single-mode fiber forms a macro-bend, WGMs may be created, propagating in the cladding or buffer. These WGMs can interfere with the guided core mode to produce interference induced oscillations in the bend loss spectral response [5]. The dominant source of WGMs occurs at the buffer-air interface and also at the cladding-buffer interface. The formation of such whispering gallery modes effectively creates an interferometer within the fiber, with the core and buffer/cladding as the two arms. To utilize a macro-bend fiber as an edge filter, an absorption layer is applied to the buffer coating to eliminate these WG modes, which makes the bend loss spectral response smoother and ideally achieves a linear response versus wavelength [8]. The temperature sensitivity of such a fiber filter arises mainly from the characteristic properties of the buffer coating such as the thermo-optic coefficient (TOC) and thermal expansion coefficient (TEC). The TOC and TEC of the buffer coating, such as acrylates, are much higher than those of fused silica of the core and the cladding of the fiber.

Macro-bend fiber edge filters can be based on low bend loss fiber such as SMF28 fiber, or high bend loss fiber such as 1060XP. The most common single-mode fiber, SMF28 fiber, has two buffer coating layers [8], [9]. Due to the coating layers, even with the absorption layer a low level of reflection from the cladding-primary coating boundary will still exist and interfere with the core mode. As a result of this when there is a change in temperature which changes the refractive index and thickness of the buffer coating, the path length variation of the WG modes and phase difference between the WG mode and core mode 
TABLE I

PARAMETERS OF STANDARD SMF28 FIBER AND 1060XP FIBER AT $1550 \mathrm{~nm}$

\begin{tabular}{|c|c|c|c|c|}
\hline \multirow{2}{*}{ SMF28 } & Core & Cladding & \multirow[t]{2}{*}{ Buffer coating 1} & \multirow[t]{2}{*}{ Buffer Coating 2} \\
\hline & & & & \\
\hline $\operatorname{Radius}(\mu \mathrm{m})$ & 4.15 & 62.5 & 95 & 125 \\
\hline $\begin{array}{l}\text { Refractive } \\
\text { index }\end{array}$ & 1.4504 & 1.4447 & 1.4786 & 1.5294 \\
\hline $\operatorname{TEC} \alpha\left(\mathrm{K}^{-1}\right)$ & $5.5 \times 10^{-7}$ & $5.5 \times 10^{-7}$ & $800 \times 10^{-7}$ & $<100 \times 10^{-7}$ \\
\hline $\operatorname{TOC} \beta\left(\mathrm{K}^{-1}\right)$ & $1 \times 10^{-5}$ & $1 \times 10^{-5}$ & $-29 \times 10^{-4}$ & \\
\hline \multicolumn{2}{|c|}{ V(normalized Frequency) } & \multicolumn{2}{|c|}{2.1611} & \\
\hline \multicolumn{5}{|c|}{ 1060XP } \\
\hline Radius $(\mu \mathrm{m})$ & 2.65 & 62.5 & - & - \\
\hline $\begin{array}{l}\text { Refractive } \\
\text { index }\end{array}$ & 1.4631 & 1.4564 & - & - \\
\hline $\operatorname{TEC} \alpha\left(\mathrm{K}^{-1}\right)$ & $5.5 \times 10^{-7}$ & $5.5 \times 10^{-7}$ & $800 \times 10^{-7}$ & - \\
\hline $\operatorname{TOC} \beta\left(\mathrm{K}^{-1}\right)$ & $1 \times 10^{-5}$ & $1.1 \times 10^{-5}$ & $-29 \times 10^{-4}$ & - \\
\hline $\mathrm{V}($ normalized & requency) & 1.502 & & \\
\hline
\end{tabular}

leads to constructive and destructive interference between the WG mode and the core mode and leads to oscillatory variations in the spectral response of the bend loss. A macro-bend fiber filter without a buffer coating together with an absorption layer can eliminate the temperature-induced periodic variations in the bend loss. The fiber filter based on SMF28 fiber requires multiple bend turns with small bend radii to achieve a better slope and high wavelength resolution. The removal of buffer coating over a metre or more of fiber is beyond practical limits as the fiber breaks if it is wrapped for more than one turn at small bend radii without a buffer. However a fiber such as 1060XP is highly sensitive to bend effects due to its low normalized frequency $(\mathrm{V})$. The $\mathrm{V}$ parameter for 1060XP fiber is 1.5035 while for SMF28 fiber it is 2.1611. Since the normalized frequency of the 1060XP is smaller, power will be less confined in the core and will be more susceptible to bending loss and furthermore the optical energy lost from the core due to bending will be higher when compared to SMF28. Therefore an edge filter based on bend sensitive fibers such as 1060XP requires only one bend turn and hence the buffer can be stripped easily and an absorption layer applied to directly to the cladding. This configuration eliminates the different TOCs and TECs of the buffer, cladding and the core. Together with an absorption layer for the cladding which absorbs the radiated modes, prevents any possibility of a re-coupling with the core mode and the reflections caused at the interfaces. Since the cladding and core are made of silica material and have a positive thermo-optic coefficient, the thermally induced effective change in refractive index of the core and cladding is linear in nature, resulting in a linear variation of bend loss with temperature. Since the temperature-dependent loss is proportional to the bend loss in the fiber filter, 1060XP fiber shows higher temperature-induced loss, when compared to its SMF28 counterpart, both with a single turn. The removal of the buffer coating and the application of an appropriate absorption layer

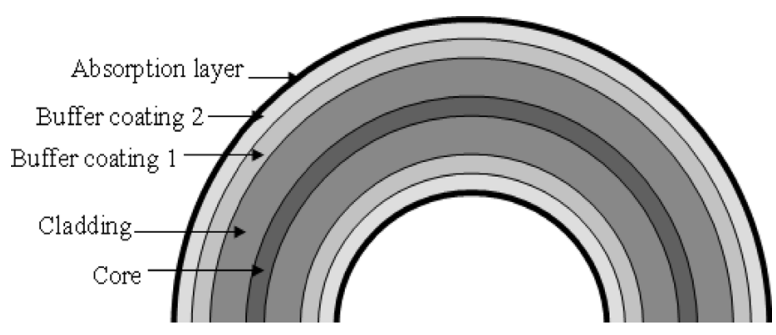

(a)

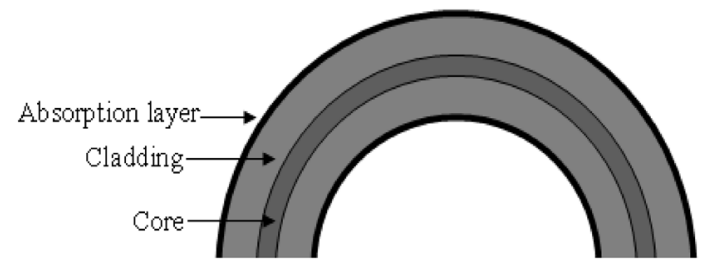

(b)

Fig. 1. Cross section view of the bend fiber in the two cases (a) with buffer and absorption layer (SMF28) and (b) without buffer and with absorption layer (1060XP).

also results in a monotonic increase in bend loss with bending radius and wavelength which is approximately equivalent to a core-infinite cladding structure [10]. For a system with this configuration temperature corrected calibration is feasible. Temperature corrected calibration means that temperature of the fiber filter is continually measured and therefore the measurement system can apply correction factors to the calibration in use. This allows the system to be used over a wide range of ambient temperatures.

Most of the edge filter based wavelength measurement systems employ a ratiometric power measurement scheme [11], [12]. A ratiometric scheme has the advantage that the system is independent of source power variations, resulting in improved system stability and accuracy. The input signal from the source splits into two equal signals, one goes to the fiber filter and the other one is the reference signal as shown in Fig. 2. The system effectively operates as a discriminator, where the ratio of the power levels reaching the photo detectors is wavelength dependent. The wavelength of the input signal can be determined, assuming a suitable calibration has taken place, using the ratio of the electrical outputs of the two photo detectors.

\section{EXPERIMENT}

Macro-bend fiber based wavelength measurement systems were built utilizing a ratiometric scheme. The following fiber filters were fabricated 1) using SMF28 fiber with the buffer retained with an absorption coating and with multiple bend turns, 2) using a single bend turn SMF28 fiber filter without a buffer and with an absorption layer, and 3) using a single bend turn 1060XP fiber filter without buffer and with an absorption layer. The absorption layer coating material used was a black Indian ink which is considered as a good optical absorber [13]. The input source used was a tunable laser source with a wavelength range from $1500 \mathrm{~nm}$ to $1600 \mathrm{~nm}$. The power stability of the laser source was $\pm 0.01 \mathrm{~dB}$ for $0 \mathrm{dBm}$ output power. The wavelength resolution and accuracy of the laser was $10 \mathrm{pm}$ and $\pm 10 \mathrm{pm}$ respectively at a constant temperature after 1 hour of warm-up. A 


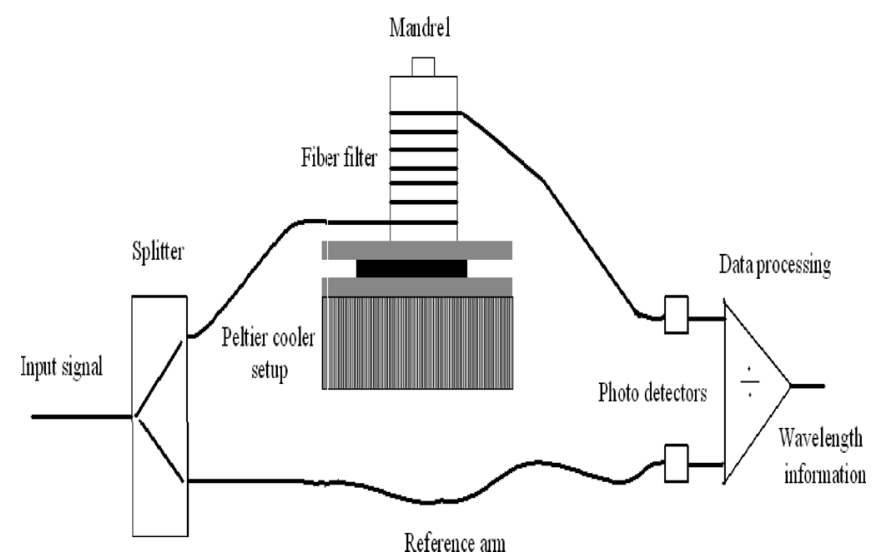

Fig. 2. Experimental arrangement to study the effect of temperature on wavelength measurement.

commercially available fused $3 \mathrm{~dB}$ coupler is used to split the input signal into two, in which one goes to the macro-bend fiber filter and the other serves as the reference signal. High sensitivity photodiodes are used at the receiver end to allow measurement of the power ratio and thus yield the wavelength information. Fig. 2 shows the schematic of the experimental setup used to study the influence of temperature on the wavelength measurements.

In the case of the SMF28 fiber filter, the fiber was wrapped over around a mandrel of fixed radius which is fixed to the metal plate base. The metal plate base was connected to the Peltier cooler, which was driven by a temperature controller and with the temperature of the fiber filter accurately monitored. The bend radius of the SMF28 fiber filter was fixed at $10.5 \mathrm{~mm}$ [1]. The discrimination range of the system can be varied by changing the number of bend turns. For the SMF28 fiber, three filters were tested with 5,10 , and 15 bend turns.

For the 1060XP fiber filter since it requires only one bend turn, the fiber filter is directly fixed to the metal plate, instead of being wrapped around a mandrel. The bend radius of the 1060XP fiber filter was fixed at $11 \mathrm{~mm}$. The calibration ratio responses of both the systems were obtained at $20^{\circ} \mathrm{C}$. The variation in ratio from the calibrated response and the corresponding wavelength variation were measured for a temperature range of $0^{\circ} \mathrm{C}$ to $60^{\circ} \mathrm{C}$ with an interval of $2{ }^{\circ} \mathrm{C}$. As the temperature controller requires time to settle down, and also for allowing a uniform distribution of the temperature within the fiber filter, the individual measurements were taken at 5 minute time intervals. The results are discussed in the next section.

\section{RESULTS AND DISCUSSION}

\section{A. SMF28 Fiber Filter Based System}

The ratio response measured for the system based on SMF28 fiber with an absorption layer for all three filters with 5, 10, and 15 bend turns and with bend radius $10.5 \mathrm{~mm}$ is shown in Fig. 3 . The fiber filter with 15 turns provides a useful discrimination slope while also covering a wavelength range $90 \mathrm{~nm}$ between $1500 \mathrm{~nm}$ to $1600 \mathrm{~nm}$ with an average slope of $0.22 \mathrm{~dB} / \mathrm{nm}$. Further increases in the number of bend turns affects the linearity of

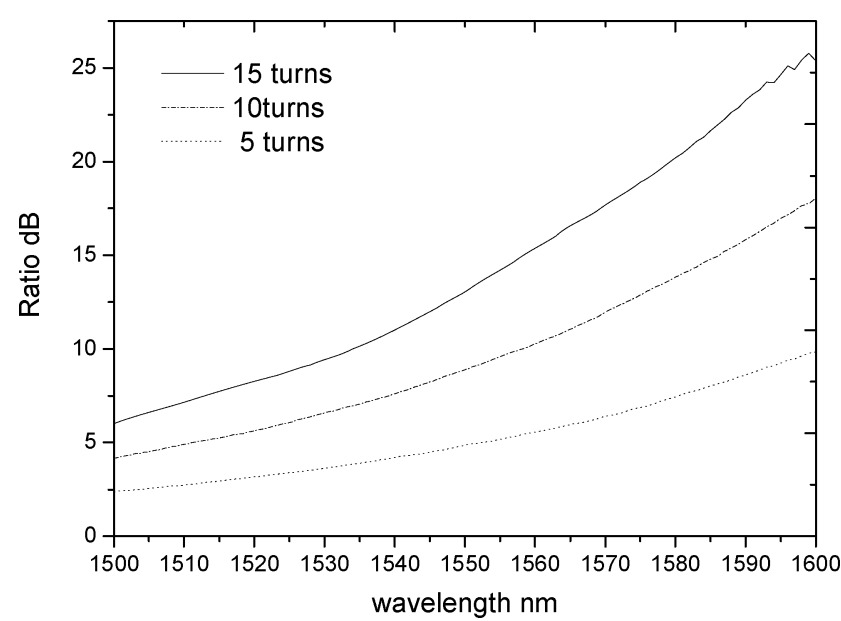

Fig. 3. Ratio response of the system with SMF28 fiber filter with different number of bend turns.

the ratio response at higher wavelengths and reduces the measurable wavelength range due to the limited SNR of the source [14]. Filters with 5 and 10 turns give a $100 \mathrm{~nm}$ wavelength range with an average slope of $0.075 \mathrm{~dB} / \mathrm{nm}$ and $0.14 \mathrm{~dB} / \mathrm{nm}$, respectively.

To investigate the influence of temperature variation on the ratio response we measured the change in ratio of the system from the calibrated response (obtained at $20^{\circ} \mathrm{C}$ ) for all the filters at $1550 \mathrm{~nm}$ for the temperature range from $0^{\circ} \mathrm{C}$ to $60^{\circ} \mathrm{C}$. The results are presented in Fig. 4(a). From the figure it is clear that the absorption layer did not eliminate the WG modes completely and the remaining WG modes produce reflections at the cladding-primary coating boundary. This results in an oscillatory nature for the variation in bend loss and the same for the ratio of the system. The total bend length is high for a larger number of bend turns resulting in stronger WGM effects and hence higher amplitude oscillations in the ratio of system. This variation in ratio from the calibrated response will induce wavelength inaccuracy. The temperature-induced wavelength variation measured at $1550 \mathrm{~nm}$ is shown in Fig. 4(b). From the figure we can see that the wavelength variations for the systems with all three filters are similar. This is due to the difference in slope of each filter, i.e., for the same ratio variation in the case of a system with a filter of 5 turns there is a large wavelength variation, while for a system with a filter of 15 turns, which has a higher slope, there is a smaller wavelength variation. Thus even though the temperature-induced ratio variation is higher for systems with a larger number of turns, due to the difference in ratio slope of the system, all systems will produce similar wavelength measurement variations. As a result, a reduction in the number of turns for the filter will not eliminate the temperature-induced wavelength error even though it reduces the amplitude of temperature-induced ratio oscillations.

It is known that the relative phase of the WG mode and the core mode is also dependent on the wavelength of the light used [4]. To understand the complexity in the temperature calibration procedure, temperature-induced ratio variation is measured at different wavelengths. The measured ratio variation of the system with a fiber filter with 15 bend turns at wavelengths 1530 


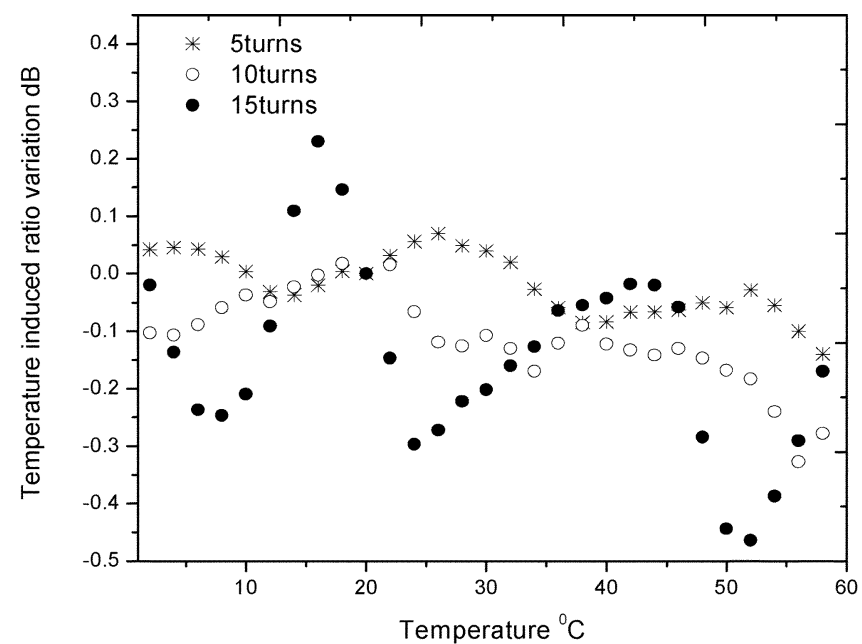

(a)

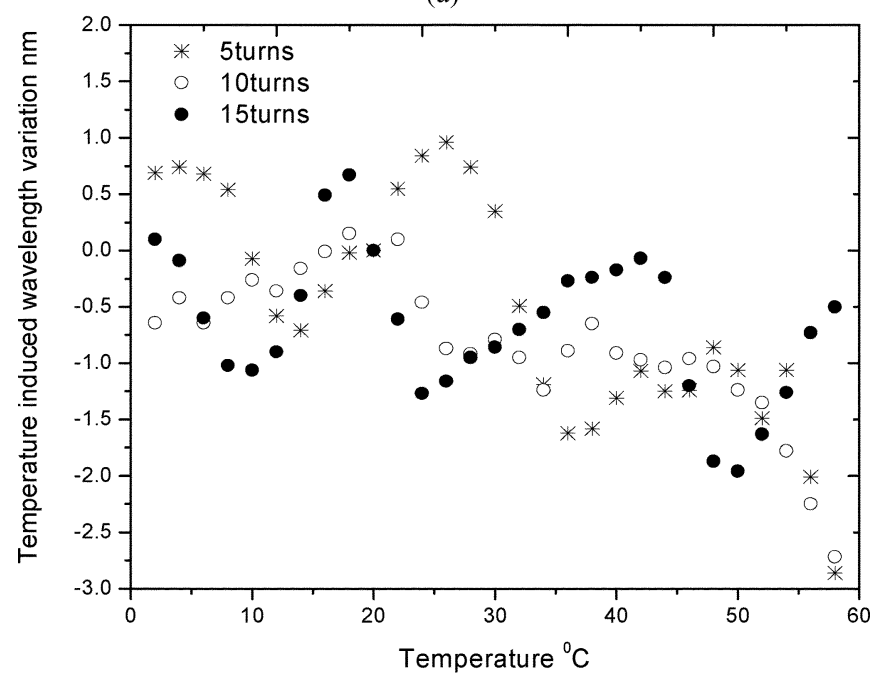

(b)

Fig. 4. Temperature-induced variation at 1550 for SMF28 fiber filter based system with 15 bend turns and $10.5 \mathrm{~mm}$ radius. (a) Variation in ratio. (b) Variation in wavelength.

$\mathrm{nm}$ and $1550 \mathrm{~nm}$ is shown in Fig. 5. At lower wavelengths since the bend loss is low, the temperature-dependent bend loss and ratio will also be smaller. It is clear from the figure that for the same filter the temperature-induced oscillations are different at different wavelengths. Thus in the case of systems based on the SMF28 fiber filter with a fiber buffer and absorption layer, a correction in the calibrated ratio response to compensate the temperature-induced ratio and wavelength variation is too complex to be feasible.

A correction of the calibration of the ratio of the system to take account of the temperature change is possible only when there is linear change in ratio with temperature for a fixed bend radius and number of turns. To achieve this, the filter has to be buffer stripped and coated with an absorption layer. Since the standard SMF28 fiber requires multiple turns to achieve a large discrimination range, winding the fiber without buffer is a complex task. To illustrate this, the ratio response of a single turn fiber filter with SMF28 fiber, without buffer and with an absorption layer is measured and is shown in Fig. 6(a) while the ratio variation at $1550 \mathrm{~nm}$ when temperature changes from $0^{\circ} \mathrm{C}$ to

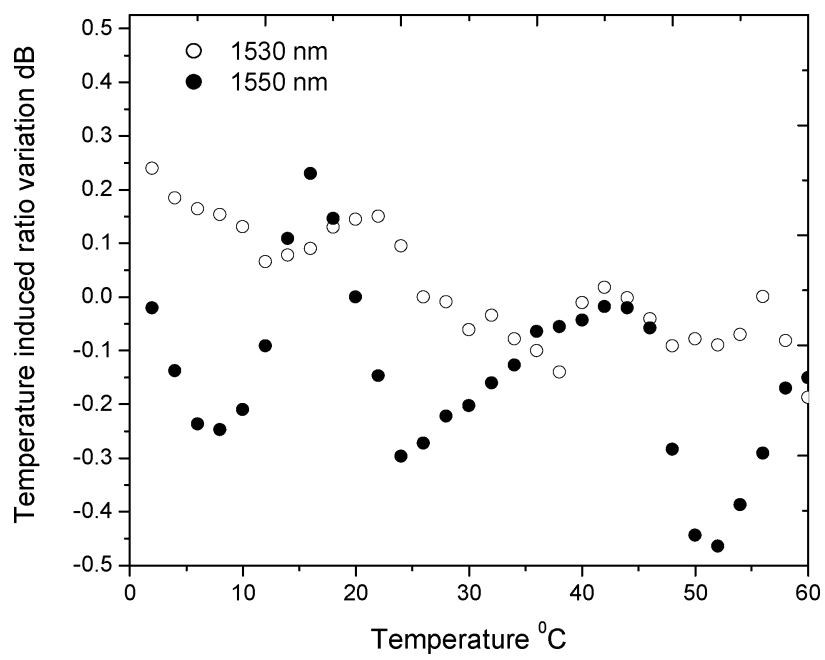

Fig. 5. Temperature-induced ratio variation at different wavelengths.

$60^{\circ} \mathrm{C}$ is shown in Fig. 6(b). From the figure it is clear that the oscillatory behavior is eliminated by removing the buffer, which allows a correction to the calibrated ratio response for a temperature-induced variation, but a filter with such a small slope $(0.016 \mathrm{~dB} / \mathrm{nm})$ is not suitable for wavelength measurements as it would severely limit the measurement resolution.

\section{B. 1060XP Fiber Filter Based System}

The use of a high bend loss fiber (such as 1060XP) eliminates the requirement of multiple bend turns to achieve larger discrimination ranges. For the 1060XP fiber, a bend radius of 11 $\mathrm{mm}$ with one bend turn gives a slope approximately equal to 10 turns of SMF28 fiber. Since it requires only one bend turn, the removal of the buffer coating is possible in the case of 1060XP fiber. The ratio response of the system at $20^{\circ} \mathrm{C}$ with a bend radius of $11 \mathrm{~mm}$ and one bend turn, with an applied absorption layer to absorb the WG modes, is shown in Fig. 7 which covers the wavelength range from $1500 \mathrm{~nm}$ to $1600 \mathrm{~nm}$ with an average slope of $0.122 \mathrm{~dB} / \mathrm{nm}$.

From the experiments conducted with SMF28 fiber filter, it was clear that removing the buffer and applying an absorption layer gives a monotonic variation in the ratio with temperature at a fixed wavelength and bend radius. The temperature-induced ratio variation for the $1060 \mathrm{XP}$ fiber filter system is shown in Fig. 8(a) when the temperature changes from $0^{\circ} \mathrm{C}$ to $60^{\circ} \mathrm{C}$ for a range of wavelengths from $1500 \mathrm{~nm}$ to $1600 \mathrm{~nm}$ at $10 \mathrm{~nm}$ intervals. The 1060XP fiber filter without a buffer provides a linear change in the ratio with temperature. The result shows that even though the ratio variation of the system is linear for the 1060XP fiber filter, the filter is highly temperature dependent. The difference between the measured wavelengths and actual wavelengths for $1500 \mathrm{~nm}, 1530 \mathrm{~nm}, 1550 \mathrm{~nm}$, and $1570 \mathrm{~nm}$ due to temperature variation is shown in Fig. 8(b). From the figure, it is clear that at higher wavelengths the temperature-dependent ratio and wavelength error is higher than for lower wavelengths. This results in larger inaccuracies at higher wavelength regions than in lower wavelength regions for a same temperature variation.

However, regardless of the high-temperature dependency of the 1060XP fiber filter, the linear variation in the ratio means 


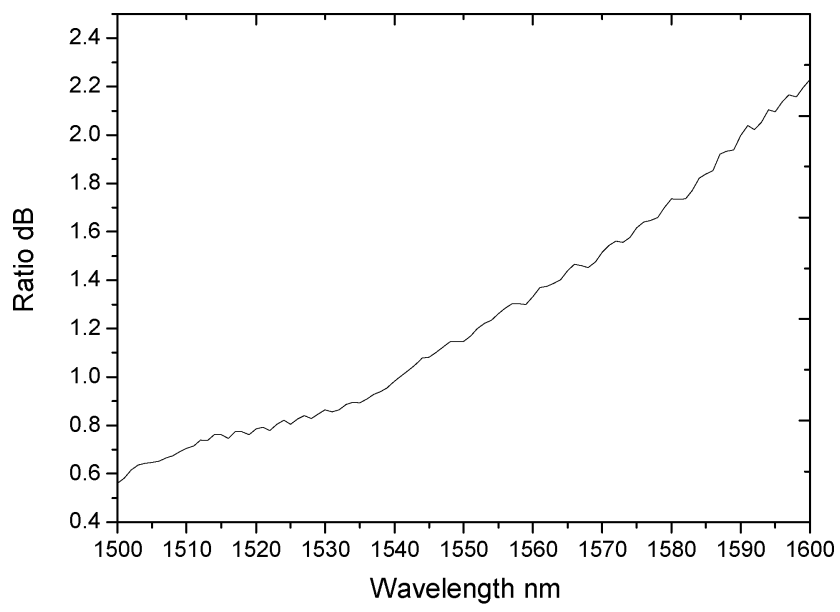

(a)

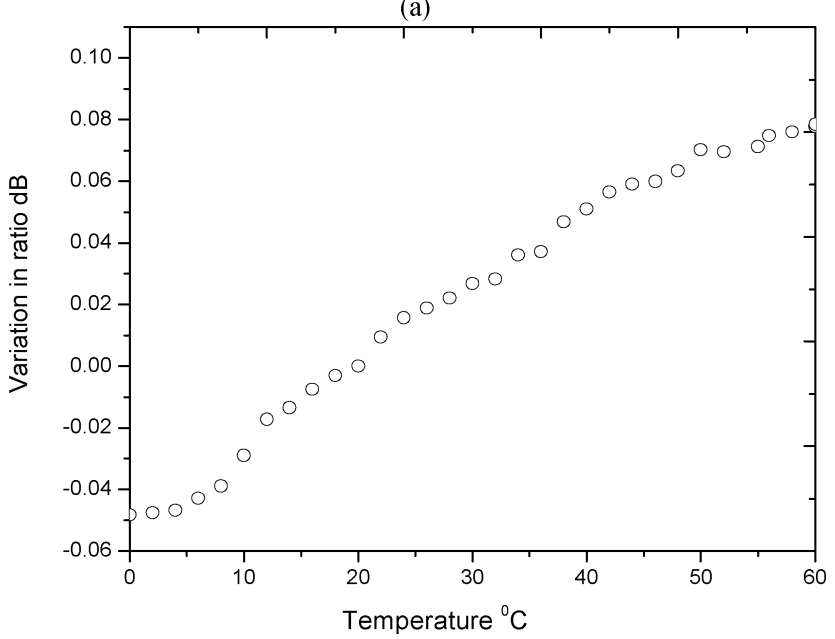

(b)

Fig. 6. (a) Ratio response of the system with one turn of SMF28 fiber filter without buffer and with absorption layer. (b) Ratio variation at $1550 \mathrm{~nm}$ when temperature varies from $0{ }^{\circ} \mathrm{C}$ to $60^{\circ} \mathrm{C}$.

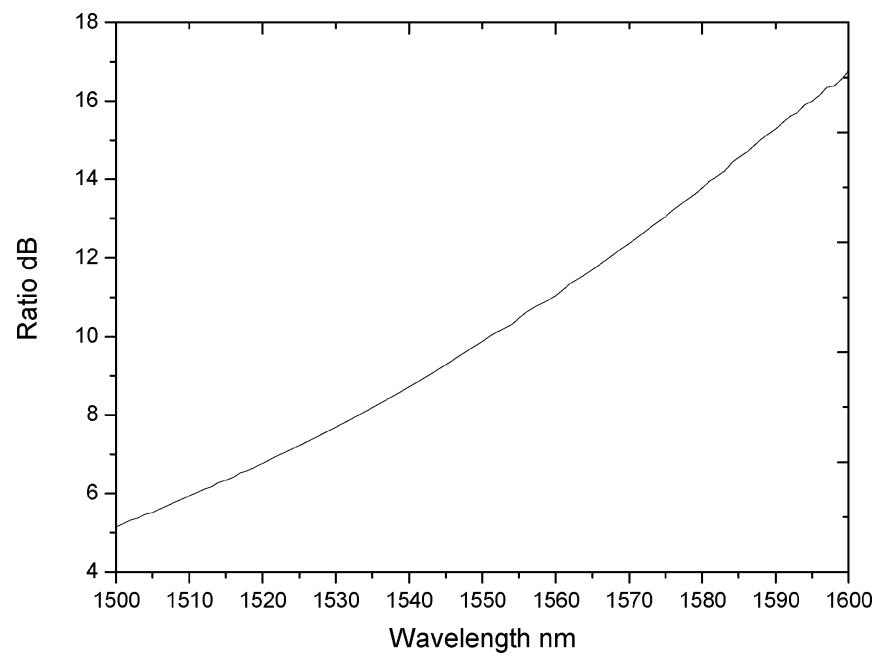

Fig. 7. Ratio response of the $1060 \mathrm{XP}$ fiber filter with radius $11 \mathrm{~mm}$ and one bend turn.

it is feasible to apply calibration correction factors to minimize the temperature-induced errors. Fig. 9 shows the required correction for the calibrated response at different temperatures. The

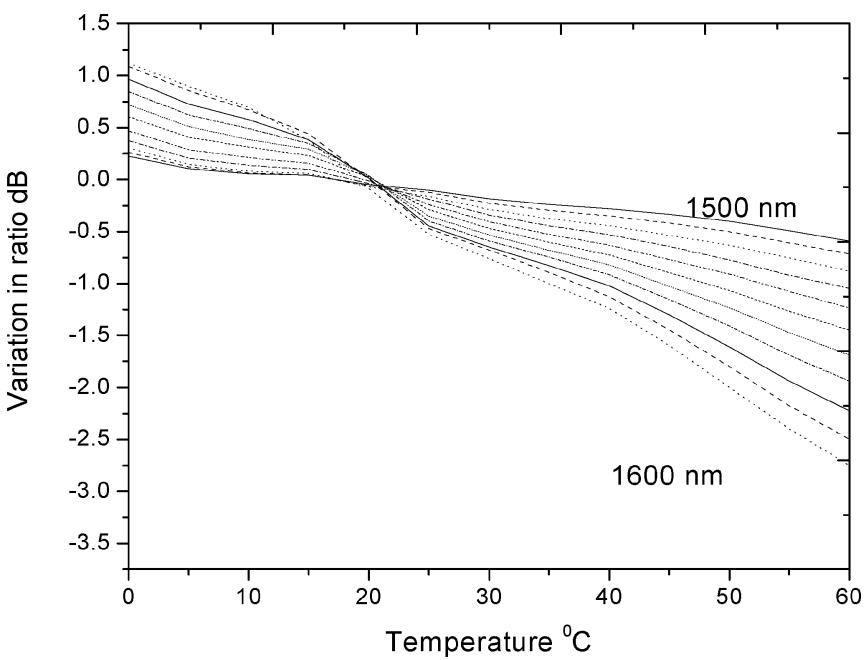

(a)

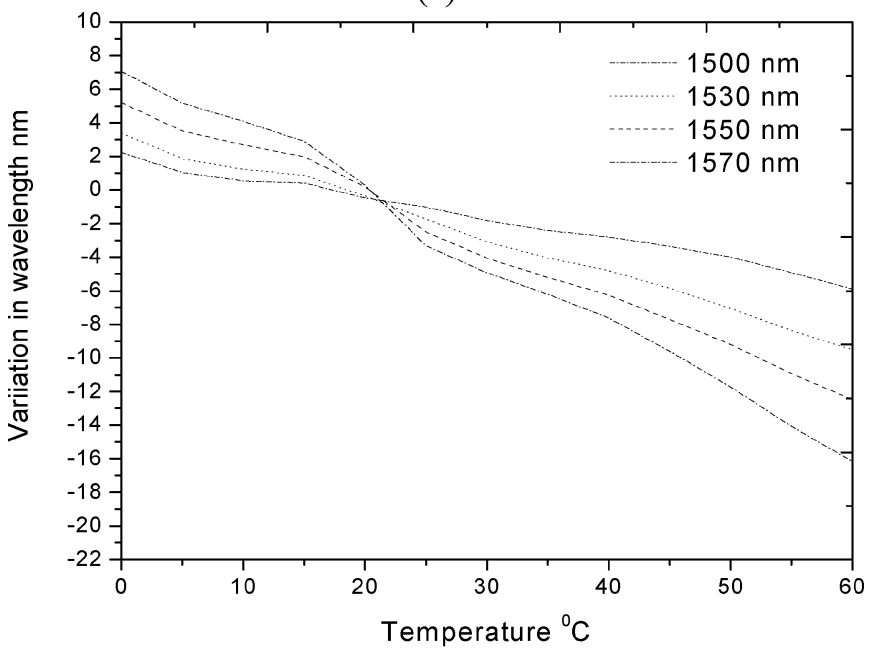

(b)

Fig. 8. Temperature-induced variation of the system based on 1060XP fiber filter. (a) Variation in ratio. (b) Variation in different wavelengths.

required correction for the ratio response is calculated for different temperatures with an interval of $5^{\circ} \mathrm{C}$ in the range from $0^{\circ} \mathrm{C}$ to $60^{\circ} \mathrm{C}$. By monitoring the temperature of the filter itself and by applying an appropriate correction to the calibration response, precise wavelength measurements can be obtained with a system based on 1060XP fiber filter, even with significant ambient temperature changes.

\section{Comparison Between SMF28 Fiber Filter and 1060XP Fiber Filter Based System}

A comparison of the wavelength measurement system based on SMF28 fiber filter with a buffer and an absorption layer and the 1060XP fiber filter without a buffer and with an absorption layer is shown in Table II for a wavelength of $1550 \mathrm{~nm}$. From the table it can be seen that the wavelength error is less for system with SMF28 fiber filter. The maximum measured wavelength variation from the wavelength $1550 \mathrm{~nm}$ for the system with an SMF28 fiber filter with 15 turns was $+0.67 \mathrm{~nm}$ at $18^{\circ} \mathrm{C}$ as the temperature decreases towards $0{ }^{\circ} \mathrm{C}$ and $-1.96 \mathrm{~nm}$ at $50^{\circ} \mathrm{C}$ as the temperature increases towards $60^{\circ} \mathrm{C}$. For the system with 
TABLE II

COMPARISON OF THE TEMPERATURE DEPENDENCY OF THE WAVELENGTH MEASUREMENT System WITH SMF28 FIBER FILTER AND 1060XP FIBER FILTER AT 1550 nm

\begin{tabular}{|c|c|c|c|c|c|c|c|}
\hline $\begin{array}{l}\text { Type of macro- } \\
\text { bend fiber filter }\end{array}$ & $\begin{array}{l}\text { Radius } \\
\mathrm{mm}\end{array}$ & $\begin{array}{l}\text { No. of } \\
\text { Bend } \\
\text { turns }\end{array}$ & $\begin{array}{l}\text { Temperature } \\
\text { change }{ }^{0} \mathrm{C} \\
\left(\text { cal. temp }-20{ }^{\circ} \mathrm{C}\right)\end{array}$ & $\begin{array}{l}\text { Max. ratio } \\
\text { change } \\
(\mathrm{dB})\end{array}$ & $\begin{array}{l}\text { Wavelength change } \mathrm{nm} \\
\text { (Central wavelength- } \\
1550 \mathrm{~nm})\end{array}$ & $\begin{array}{l}\text { Nature of } \\
\text { variation }\end{array}$ & $\begin{array}{l}\text { Correction for } \\
\text { the calibrated } \\
\text { ratio response }\end{array}$ \\
\hline $\begin{array}{l}\text { SMF28 } \\
\text { with buffer and } \\
\text { absorption layer }\end{array}$ & 10.5 & 15 & $0-60$ & $0.464 @ 50{ }^{0} \mathrm{C}$ & $\begin{array}{l}1550-1.96 \mathrm{~nm} @ 50{ }^{0} \mathrm{C} \\
(1550.67 \mathrm{~nm}-1548.04 \mathrm{~nm})\end{array}$ & Oscillatory & Very complex \\
\hline $\begin{array}{l}1060 X P \text { without } \\
\text { buffer and with } \\
\text { absorption layer }\end{array}$ & 11 & 1 & $0-60$ & $2.05 @ 60{ }^{0} \mathrm{C}$ & $\begin{array}{l}1550-12.4 \mathrm{~nm} @ 60^{\circ} \mathrm{C} \\
(1555.2 \mathrm{~nm}-1537.6 \mathrm{~nm})\end{array}$ & $\begin{array}{l}\text { Approx. } \\
\text { linear }\end{array}$ & Yes \\
\hline
\end{tabular}

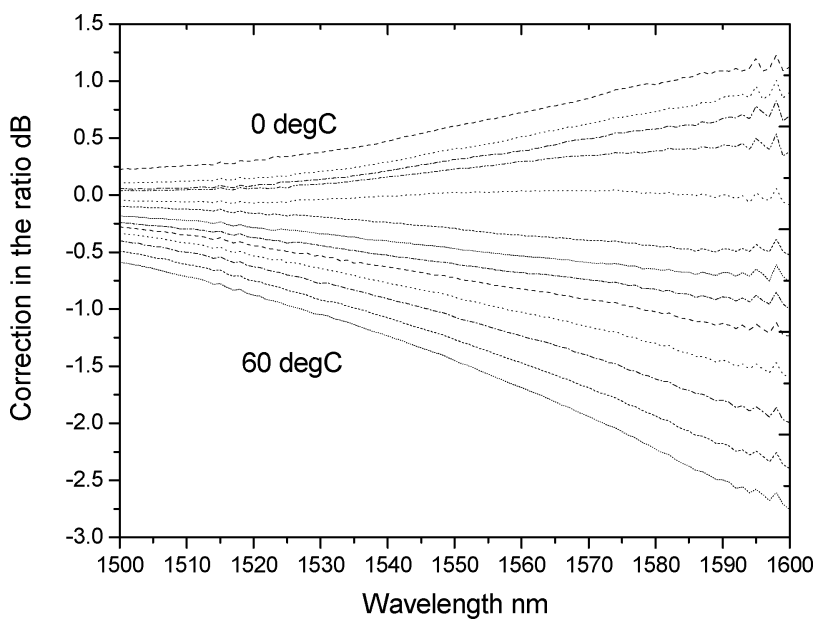

Fig. 9. Required correction for the calibrated ratio response at different temperatures.

1060XP fiber filter the wavelength variation was $+5.2 \mathrm{~nm}$ as the temperature approaches $0^{\circ} \mathrm{C}$ and $-12.4 \mathrm{~nm}$ as the temperature increases towards $60^{\circ} \mathrm{C}$. While it is apparent that the SMF28 fiber filter based system is less temperature sensitive, nevertheless the oscillatory nature of bend loss and ratio of the system makes correction of the calibrated response unfeasible. For the SMF28 based filter the only option is to use active temperature stabilization of the filter temperature. Whereas for a 1060XP based filter temperature compensation requires a sensor and compact electronics only, temperature stabilization will additionally demand a Peltier cooler, two heat sinks, a complex feedback control system and, depending on the ambient temperature variation to be dealt with, will involve significantly higher power consumption by the system. The temperature stabilization approach will thus require more physical space, as well as higher complexity and cost than the temperature compensation approach. The 1060XP fiber filter has a higher temperature dependence than the SMF28 fiber filter, but due to the linear nature of the ratio variation with temperature, the temperature-induced error can be compensated for by adding correction factors to the calibration ratio response. The wavelength accuracy can be improved by obtaining the correction in the ratio response with smaller temperature intervals or extrapolating the correction response between the required temperature intervals. Thus irrespective of temperature dependence of the 1060XP fiber filter it can be operated over a wide temperature range, if the correction in ratio response is added to the original ratio response and precise wavelength measurements can be obtained.

\section{CONCLUSION}

The effect of temperature on a wavelength measurement system based on macro-bend fiber filter is presented. From the results it is shown that for a fiber filter, without a buffer, and with an absorption layer, the oscillatory behavior of the ratio variation with temperature, which results from the interference between the WG modes and the core mode can be eliminated. For an SMF28 fiber filter, which has a dual coating layer, the ratio and wavelength variation is oscillatory in nature and different for different wavelengths and this makes the temperature corrected calibration approach too complex to be feasible. For an SMF28 based system temperature stabilization is the only option, with a consequent increase in overall system complexity and cost given the need for an active Peltier cooler temperature control system. For a 1060XP fiber filter without a buffer and with an absorption layer the system produces a linear ratio variation. While the SMF28 fiber filter based system is less sensitive to temperature than the 1060XP fiber filter based system, nevertheless the linear nature of the temperature dependence of 1060XP fiber filter means that it is feasible to correct for changes in ambient temperature by monitoring the filter temperature and correcting the calibration response. Thus a 1060XP filter based system can be operated over a wide ambient temperature range for precise wavelength measurement. For a system based in SMF28 filter active temperature stabilization of the filter will be required to achieve the same result.

\section{REFERENCES}

[1] Q. Wang, G. Farrell, T. Freir, G. Rajan, and P. Wang, "Low-cost wavelength measurement based on a macrobending single-mode fiber," Opt. Lett., vol. 31, no. 12, pp. 1785-1787, 2006.

[2] Q. Wang, G. Rajan, P. Wang, and G. Farrell, "Macrobending fiber loss filter, ratiometric wavelength measurement and application," Meas. Sci. Technol., vol. 18, pp. 3082-3088, 2007.

[3] R. Morgan, J. S. Barton, P. G. Harper, and J. D. C. Jones, "Wavelength dependence of bending loss in monomode optical fibers: Effect of buffer coating," Opt. Lett., vol. 15, no. 17, pp. 947-949, 1990.

[4] F. M. Haran, J. S. Barton, S. R. Kidd, and J. D. C. Jones, "Optical fiber interferometric sensors using buffer guided light," Meas. Sci. Technol., vol. 5, pp. 526-530, 1994.

[5] R. Morgan, J. S. Barton, P. G. Harper, and J. D. C. Jones, "Temperature dependence of bending loss in monomode optical fibers," Electron. Lett., vol. 26, no. 13, pp. 937-939, 1990.

[6] S. H. Nam and S. Yin, "High temperature sensing using whispering gallery mode resonance in bent optical fibers," IEEE Photon. Technol. Lett., vol. 17, no. 11, pp. 2391-2393, Nov. 2005.

[7] P. Wang, G. Farrell, Q. Wang, and G. Rajan, "An optimized edge filter for wavelength measurements," IEEE Photon. Technol. Lett., vol. 19, no. 15 , pp. 1136-1138, 2007. 
[8] Q. Wang, G. Farrell, and T. Freir, "Theoretical and experimental investigations of macro-bend losses for standard single mode fibers," Opt. Expr., vol. 13, pp. 4476-4484, 2005.

[9] Q. Wang, G. Rajan, P. Wang, and G. Farrell, "Polarization dependence of bend loss for a standard singlemode fiber," Opt. Expr., vol. 15, no. 8, pp. 4909-4920, 2007.

[10] D. Marcuse, "Curvature loss formula for optical fibers," J. Opt. Soc. Amer., vol. 66, no. 3, pp. 216-220, 1976.

[11] Y.Zhao and Y. Liao, "Discrimination methods and demodulation techniques for fiber Bragg grating sensors," Opt. Lasers Eng., vol. 41, pp. $1-18,2004$.

[12] S. M. Melle, K. Liu, and R. M. Measures, "A passive wavelength demodulation system for guided Bragg grating sensors," IEEE Photon. Technol. Lett., vol. 4, pp. 516-518, Apr. 1992.

[13] J. R. Mourant, I. J. Bigio, D. A. Jack, T. M. Johnson, and H. D. Miller, "Measuring absorption coefficients in small volumes of highly scattering media: source-detector separations for which path lengths do not depend on scattering properties," Appl. Opt., vol. 36, no. 22, pp. 5655-5661, 1997.

[14] Q. Wang, G. Farrell, and T. Freir, "Study of transmission response of edge filters employed in wavelength measurements," Appl. Opt., vol. 44, no. 36, pp. 7789-7792, 2005.

Ginu Rajan received the B.Sc. degree in physics from the University of Kerala, India, and the M.Sc. degree in applied physics from Mahatma Gandhi University, Kerala, India, in 2000 and 2002, respectively. He worked as a Research Trainee at the Indian Institute of Astrophysics during the period 2003-2005. Since 2005 he has been working towards the Doctoral degree at the Photonics Research Centre, Dublin Institute of Technology, Dublin, Ireland. His research interests include optical fiber sensors and their applications, fiber edge filters, FBG interrogation systems, and fast wavelength measurement techniques.
Yuliya Semenova received the B.Sc. degree in electronic engineering and the Ph.D. degree in physics of molecular and liquid crystals from Lviv Polytechnic National University, Ukraine, in 1992 and 1999, respectively.

Between 1997 and 2001, she worked as a Researcher in the Faculty of Electrophysics, Lviv Polytechnic National University. Since 2001, she has been a Researcher and Lecturer in the School of Electronic and Communications Engineering, Dublin Institute of Technology, Dublin, Ireland. Her research interests include physics of liquid crystals and their applications in photonics and fiber optic sensing. She has published over 50 articles in journals and conferences.

Pengfei Wang has been working towards the Ph.D. degree at the Photonics Research Centre, Dublin Institute of Technology, Dublin, Ireland, since 2005. His research interests include optoelectronics devices and the modelling and experimental investigations of bend loss in waveguides and fibers.

Gerald Farrell is the Head of School of the School of Electronic and Communications Engineering at the Dublin Institute of Technology (DIT), Dublin, Ireland. He graduated with an honours degree in electronic engineering from University College Dublin in 1979 and spent a number of years as a Communications Systems Design Engineer before joining the DIT. He received the $\mathrm{Ph} . \mathrm{D}$. degree from Trinity College Dublin for research in all-optical synchronization using self-pulsating laser diodes. He is also the Principal Investigator and Director of the Photonics Research Centre at the Dublin Institute of Technology. The Centre's research concentrates on optical sensing, in particular FBG sensing, the modeling and application of bend loss in optical fiber and integrated waveguides and liquid crystal tunable filters for FBG sensor arrays. $\mathrm{He}$ is the project leader on several research projects, some with international partners. Dr. Farrell has over 100 publications in the area of photonics, holds several patents, and is a consultant for a number of well-known companies in the U.K. and Ireland. 